University of Nebraska - Lincoln

DigitalCommons@University of Nebraska - Lincoln

2019

Differential Relationship between Physical Activity and Intake of Added Sugar and Nutrient-Dense Foods: A Cross-Sectional Analysis

Karsten Koehler

Julie B. Boron

Teresa M. Garvin

Matthew R. Bice

Jeffrey R. Stevens

Follow this and additional works at: https://digitalcommons.unl.edu/psychstevens

Part of the Nutrition Commons, and the Social and Behavioral Sciences Commons

This Article is brought to you for free and open access by the Psychology, Department of at

DigitalCommons@University of Nebraska - Lincoln. It has been accepted for inclusion in Jeffrey Stevens Papers \& Publications by an authorized administrator of DigitalCommons@University of Nebraska - Lincoln. 


\title{
Differential relationship between physical activity and intake of added sugar and nutrient- dense foods: A cross-sectional analysis
}

\author{
Karsten Koehler, ${ }^{\mathrm{a}, \mathrm{b}}$, Julie B. Boron ${ }^{\mathrm{c}}$, Teresa M. Garvin ${ }^{\mathrm{d}}$, Matthew R. Bice ${ }^{\mathrm{e}}$, Jeffrey R. Stevens ${ }^{\mathrm{b}, \mathrm{f}}$

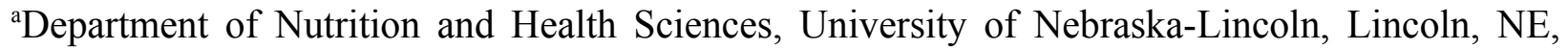 \\ USA (kkoehler3@unl.edu) \\ ${ }^{b}$ Center for Brain, Biology and Behavior, University of Nebraska-Lincoln, Lincoln, NE, USA \\ ${ }^{\mathrm{c}}$ Department of Gerontology, University of Nebraska Omaha, Omaha, NE, USA \\ (jboron@unomaha.edu) \\ ${ }^{\mathrm{d} G r e t c h e n ~ S w a n s o n ~ C e n t e r ~ f o r ~ N u t r i t i o n, ~ O m a h a, ~ N E, ~ U S A ~(t e r e s a m g a r v i n @ g m a i l . c o m) ~}$ \\ ${ }^{\mathrm{e}}$ Kinesiology and Sport Sciences Department, University of Nebraska at Kearney, Kearney, NE, \\ USA (bicemr@unk.edu) \\ fDepartment of Psychology, University of Nebraska-Lincoln, Lincoln, NE, USA \\ (jeffrey.r.stevens@gmail.com)
}

\begin{abstract}
A curvilinear relationship exists between physical activity (PA) and dietary energy intake (EI), which is reduced in moderately active when compared to inactive and highly active individuals, but the impact of PA on eating patterns remains poorly understood. Our goal was to establish the relationship between PA and intake of foods with varying energy and nutrient density. Data from the 2009-2010 United States National Health and Nutrition Examination Survey were used to include a Dietary Screener Questionnaire for estimated intakes of added sugar, fruits and vegetables, whole grains, fiber, and dairy. Participants ( $n=4766 ; 49.7 \%$ women) were divided into sex-specific quintiles based on their habitual PA. After adjustment for age, body mass index, household income, and education, intakes were compared between PA quartiles, using the lowest activity quintile (Q1) as reference. Women in the second to fourth quintile (Q2-Q4) consumed less added sugar from sugary foods ( +2 tsp/day) and from sweetened beverages $(+2$ tsp/day; all $\mathrm{p}<0.05$ vs. Q1). In men, added sugar intake was elevated in the highest activity quintile (Q5: $+3 \pm 1 \mathrm{tsp} /$ day, $\mathrm{p}=0.007$ vs. Q1). Fruit and vegetable intake increased (women: Q1-Q4 $+0.3 \pm 0.1$ cup eq/day; $\mathrm{p}<0.001$; men: Q1-Q3 $+0.3 \pm 0.1 \mathrm{cup}$ eq/day, $\mathrm{p}=0.002)$ and stagnated in higher quintiles. Dairy intake increased with PA only in men $(\mathrm{Q} 5:+0.3 \pm 0.1 \mathrm{cup}$ eq/day, $\mathrm{p}<0.001$ vs. Q1). Results demonstrate a differential relationship between habitual PA and dietary intakes, whereby moderate but not necessarily highest PA levels are associated with reduced added sugar and increased nutrient-dense food consumption. Future research should examine specific mechanisms of food choices at various PA levels to ensure dietary behaviors (i.e., increased sugary food intake) do not negate positive effects of PA.
\end{abstract}

Keywords: MET-minutes; food intake regulation; inactivity; weight status; physical activity spectrum

Published in:

Koehler, K., Boron, J.B., Garvin, T.M., Bice, M.R., \& Stevens, J.R. (2019). Differential relationship between physical activity and intake of added sugar and nutrient-dense foods: A cross-sectional analysis. Appetite, 120, 91-97. doi:10.1016/j.appet.2019.05.010 


\section{Introduction ${ }^{1}$}

Approximately two out of three Americans are overweight or obese (1), and an inactive lifestyle coupled with excessive food consumption are considered primary drivers of this obesity pandemic (2). While the beneficial effects of physical activity (PA) on many obesity-related diseases such as diabetes, cardiovascular disease, and certain cancers are well established (3-5), the effectiveness of PA on weight status, and particularly its capacity to promote weight loss, remains under debate (6). Energy balance, and ultimately weight loss and weight gain, is a direct result of dietary energy intake and energy expenditure $(7,8)$. As PA represents the only component of energy expenditure that is driven directly by behavior and therefore is its most variable contributor (9), it is critical to understand the impact of PA on food intake and particularly whether increased PA invokes a compensatory effect on food intake which could negate beneficial effects of PA on weight status (10).

It has previously been hypothesized that PA can improve appetite control, satiety signaling, and food intake $(11,12)$. However, the relationship between PA and EI seems to be non-linear (12). Mayer et al. first reported in 1956 that the relationship between habitual PA and daily EI in industrial workers was curvilinear (13), whereby EI declined as activity levels increased from inactive to moderate and then increased again towards the upper end of the PA spectrum. This Jshaped relationship has since been reintroduced and reproduced in several distinct trials. For example, data from the National Weight Control Registry showed that EI was about 4-5\% lower among moderately active individuals when compared to individuals in the lowest and highest activity quartiles (14). Further, a systematic review combining data from 10 studies also demonstrated a robust reduction in EI at moderate-to-high activity levels when compared to low and very high activity levels (15), providing further evidence for a J-shaped relationship between habitual PA and EI. The long-term implications of this curvilinear relationship for weight management were recently underscored by a prospective trial demonstrating the higher EI in individuals with the lowest activity levels resulted in a 2.5 to 3.8 -fold increased risk for unhealthy weight gain, whereas the increased EI among highly active individuals failed to cause significant weight change over the course of a one-year period (16).

While the above named studies have focused on the relationship between PA and EI, the impact of PA on more fine-grained measures of eating patterns (food choice and frequency) and nutrient intake remains only poorly understood. Cross-sectional studies in young adults suggest that

\footnotetext{
${ }^{1}$ Abbreviations

DSQ - Dietary Screener Questionnaire

EI - Energy Intake

GLM - General Linear Model

GPAQ - Global Physical Activity Questionnaire

MEC - Mobile Examination Center

METs - Metabolic Equivalents

METmin - MET-minutes

NHANES - National Health and Nutrition Examination Survey

PA - Physical Activity

Q - Quartile

tsp - teaspoon
} 
individuals who are physically active tend to consume more nutrient-dense, low-calorie foods, such as fruit, fruit juices, and dairy, and less energy-dense fast food (17-20). However, none of these studies directly quantified PA, making it difficult to detect potential non-linear doseresponse relationships between PA and food intake. Therefore, the goal of the present study was to establish if there is a dose-response relationship between habitual PA and the eating patterns and intake of key dietary factors, such as nutrient-dense foods including whole grains, fruits and vegetables, dairy, and added sugar from sugary foods (e.g., soda, doughnuts, sugary cereal) in a nationally representative sample of the United States including a broad range of adult ages. We hypothesized that a curvilinear relationship would exist between habitual PA and added sugar from sugary foods consumption such that added sugar consumption would be elevated in the least and most active individuals when compared to moderately active individuals, mimicking the previously reported relationship between PA and EI $(15,16)$. We further hypothesized that consumption of nutrient-dense foods (foods containing vitamins, minerals, complex carbohydrates, lean protein, and healthy fats such as whole grains, fruits, vegetables, dairy) would increase as activity levels increase, supporting previous findings from smaller-scale studies comparing active vs. inactive populations (17-19), but would plateau or even decrease at the upper end of the activity spectrum due to increased intake of energy-dense, nutrient poor foods to meet higher energy requirements (21).

\section{Material and Methods}

\subsection{Study Design}

The present cross-sectional analysis included data from the 2009-2010 cycle of the National Health and Nutrition Examination Survey (NHANES), which describes PA and nutrient data from a representative sample of the United States population. The 2009-2010 cycle was chosen to include information obtained using the Dietary Screener Questionnaire (DSQ), allowing for the automated analysis of estimated intakes of key dietary factors representing food choices like fruits, vegetables, whole grains, fiber which can be linked to clear and actionable recommendations for practice, policy and researchers. These benefits were considered to outweigh the use of an older data set, particularly as dietary and physical activity patterns have only changed minimally within the past 10 years $(22,23)$. The current analysis combined DSQ information with demographic data, anthropometric measures, and PA information. The study was approved by the National Center for Health Statistics Ethics Review Board. Written informed consent was obtained from all participants.

\subsection{Participants}

The present analysis includes data from 5302 adults aged $18-69$ years. Participants with a body mass index indicative of underweight $\left(<18.5 \mathrm{~kg} / \mathrm{m}^{2} ; \mathrm{n}=95\right)$ or obesity class $3\left(>40 \mathrm{~kg} / \mathrm{m}^{2} ; \mathrm{n}=381\right)$ were excluded to minimize the inclusion of participants with an underlying eating disorder such as anorexia nervosa and binge-eating disorder (24). Participants with incomplete data $(n=60)$ were also excluded.

\subsection{Dietary Screener Questionnaire}

The DSQ was developed for inclusion in the NHANES 2009-2010 and included dietary factors of interest for the prevention of heart disease and cancer. The screener was comprised of 30 food-group items to collect information on the intake of fruits, vegetables, dairy, whole grains, 
dietary fiber, calcium, added sugar, sugar-sweetened beverages, and other foods. In the NHANES 2009-2010, the DSQ was administered by a trained interviewer at the Mobile Examination Center (MEC), who assessed the frequency of consumption over the past month for each item. Using publicly available scoring algorithms, screener responses were converted by the NHANES research team to estimates of dietary intakes for fruits and vegetables (in cups; 1 cup = $150 \mathrm{~g}$ ), fiber (in g), added sugar (in tsp; $1 \mathrm{tsp}=4 \mathrm{~g}$ ), calcium (in $\mathrm{mg}$ ), dairy (in cup equivalents; 1 cup $=240 \mathrm{~mL}$ ), and whole grains (in ounce equivalents; 1 ounce $=28 \mathrm{~g}$ ), which were downloaded from the NHANES website. Conversion factors for equivalents for dairy (1 cup equivalent $=1$ cup of milk, yogurt, or fortified soymilk, 1.5 ounces of natural cheese or 2 ounces of processed cheese) and whole grains ( 1 ounce equivalent $=1$ ounce dry whole grain pasta or rice, 1 medium (1 ounce) slice whole grain bread, 1 ounce ready-to-eat whole grain cereal, 0.5 cups of cooked whole grain rice, pasta, or cereal or about 1 cup of flaked cereals) were as described in the 2015 Dietary Guidelines for Americans (25). To our knowledge, most studies reporting on the relationship between PA and food intake assessed food intake via diet recalls $(13,16)$ or food frequency questionnaires $(17,19,20,26)$. In an effort to be less costly and burdensome when compared to dietary recalls and records $(27,28)$, the DSQ provided a unique opportunity in NHANES history to ask about consumption of food, beverages, or food groups of particular interest to this study to be used as indicators of particular aspects of diet (29). Although dietary recalls are not considered sufficiently accurate for the assessment of food and nutrient intakes in individuals (30), these brief assessments provide a short, relatively simple, and valid way to rank individuals with regard to consumption of certain foods and nutrients $(31,32)$. The DSQ has recently been shown to provide intake estimates in close agreement $(<2 \%)$ with intakes obtained from two non-consecutive 24-h dietary recalls providing confidence in scale reliability (33). The DSQ also allows for analysis of the estimated intake of added sugar specifically from sugary foods and beverages rather than sugar from all foods consumed, like breads, condiments and sauces, fats and oils. It was anticipated that the DSQ would enhance the understanding of self-perceived eating patterns, specifically the frequency of consumption of sugary foods and beverages versus the traditional two-day dietary recall.

\subsection{Physical Activity}

Habitual PA was collected using the Physical Activity Questionnaire, which is based on the Global Physical Activity Questionnaire (GPAQ (34)). The GPAQ collects information on PA over the past 7 days in three settings (activity at work, travel to and from places, recreational activities) as well as sedentary behaviors and was conducted as part of the household interview. Previous validation studies have reported satisfactory agreement between accelerometry, the currently preferred objective approach to assess PA (35), and the GPAQ, as around $80 \%$ of individuals are classified similarly (36), indicating appropriate validity for the purpose of the present large-scale cross-sectional analysis. PA data were subsequently converted to energy expenditure using Metabolic Equivalents (METs), whereby 1 MET represents the energy expenditure at rest, or approximately $1 \mathrm{kcal}$ per kg body weight per hour, in adult men and women (37). Using established coefficients of 4 METs for time spent in moderate activities as well as for time spent walking and bicycling and 8 METs for time spent in vigorous activities (38), physical energy expenditure was calculated as the product of time spent (minutes per week) and the respective MET factor to obtain habitual MET-minutes (METmin). Energy expenditure in MET-minutes was calculated separately for work activities, walking and bicycling, and recreational activities; total PA expenditure was calculated as the sum of MET-minutes for these 
three domains. Based on their total MET-minutes, participants were divided into quintiles. To account for potential sex differences in habitual PA (39), quintiles were established separately for men and women.

\subsection{Additional Assessments}

Anthropometric measures were collected by trained health technicians at the MEC. Demographic characteristics, including age, sex, education, and socioeconomic status were collected at the participant homes via interviewer-administered questionnaires.

\subsection{Calculations and Statistical Analyses}

Statistical analyses were conducted with $\mathrm{R}$ version 3.3.2, The R Foundation for Statistical Computing. General linear model (GLM) analyses were used to examine the relationship between PA and dietary factor intake, using the lowest activity quintile (Q1) as reference quintile and controlling for the effects of the following confounding variables: age (under/over 55 years), BMI (overweight, obesity levels 1-2), education level, and household income (under/over \$20,000). GLM analyses were conducted separately for male and female participants. GLM results are reported as coefficient estimates \pm coefficient standard errors as well as corresponding p-values indicating the statistical significance for differences between each quintile and the reference quintile (Q1). In addition, effect sizes (Cohen's $d$ ) were calculated as the standardized mean difference between quintiles and the reference quintile by dividing estimated differences by the residual standard deviation of the GLM, and effects were considered small, medium and large for $\mathrm{d}>0.2, \mathrm{~d}>0.5$ and $\mathrm{d}>0.8$, respectively . Statistical significance was set a priori at $\mathrm{p}<$ 0.05 .

\section{Results}

\subsection{Data Set}

The final data set (Table 1) included a total of 4766 participants (2368 women, 2398 men). Approximately $27 \%$ of women did not document any PA at all (0 METmin/week), resulting in a larger sample size for Q1 $(n=647)$ when compared to the other quintiles (between $n=349$ and $\mathrm{n}=476$ ). In men, sample size was very similar among all quintiles (between $n=470$ and $n=487$ ). As men were almost twice as active when compared to women ( $4978 \pm 117$ vs. $2412 \pm 118$ $\mathrm{METmin} /$ week, $<<0.001$; equivalent to $89 \pm 2 \mathrm{vs} .43 \pm 2 \mathrm{~min} / \mathrm{d}$ of vigorous PA or $178 \pm 4 \mathrm{vs} .86$ $\pm 4 \mathrm{~min} / \mathrm{d}$ of moderate PA), quintile METmin cut points were considerably lower in women than in men. Overall, younger age was significantly associated with placement in an increasing activity quintile for both men and women (both $\mathrm{p}<0.001$ ), and higher activity levels were associated with a lower body mass index in both sexes $(\mathrm{p}<0.001)$. Household income and education demonstrated a curvilinear relationship with PA such that the highest average income and the highest average degree level occurred at moderate to high PA levels (women: Q4; men: Q3) before declining again towards the highest activity quintiles. 


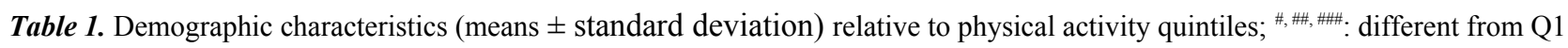
( $p<0.05, \mathrm{p}<0.01, \mathrm{p}<0.001$, respectively). $\dagger: 840 \mathrm{METmin} / \mathrm{week}=30 \mathrm{~min}$ of moderate PA or $15 \mathrm{~min} / \mathrm{d}$ of vigorous PA

\begin{tabular}{|c|c|c|c|c|c|}
\hline & \multicolumn{5}{|c|}{ Women } \\
\hline & $\begin{array}{c}\mathrm{Q} 1 \\
(\mathrm{n}=647)\end{array}$ & $\begin{array}{c}\text { Q2 } \\
(n=349)\end{array}$ & $\begin{array}{c}\text { Q3 } \\
(n=426)\end{array}$ & $\begin{array}{c}\mathrm{Q} 4 \\
(\mathrm{n}=476)\end{array}$ & $\begin{array}{c}\text { Q5 } \\
(\mathrm{n}=470)\end{array}$ \\
\hline $\begin{array}{l}\text { Physical Activity } \\
\text { (METmin/week) } \dagger\end{array}$ & $0 \pm 0$ & $296 \pm 132^{\#}$ & $875 \pm 215^{\# \# \#}$ & $2241 \pm 639^{\# \# \#}$ & $8873 \pm 6493^{\# \# \#}$ \\
\hline Age (y) & $44.9 \pm 15.0$ & $43.3 \pm 14.8$ & $41.9 \pm 14.7^{\# \#}$ & $40.3 \pm 13.9^{\# \#}$ & $40.0 \pm 14.0^{\# \#}$ \\
\hline Body Weight (kg) & $74.7 \pm 15.1$ & $73.3 \pm 14.6$ & $71.9 \pm 14.4^{\# \#}$ & $72.1 \pm 14.6^{\# \#}$ & $72.0 \pm 15.2^{\# \#}$ \\
\hline $\begin{array}{l}\text { Body Mass Index } \\
\left(\mathrm{kg} / \mathrm{m}^{2}\right)\end{array}$ & $29.1 \pm 5.2$ & $28.3 \pm 5.1^{\#}$ & 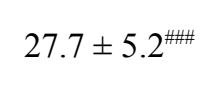 & $27.3 \pm 5.2^{\# \# \#}$ & $27.5 \pm 5.3^{\# \#}$ \\
\hline $\begin{array}{l}\text { Household Income } \\
(\$ 1000)\end{array}$ & $46.5 \pm 36.2$ & $53.4 \pm 39.0^{\#}$ & $56.5 \pm 41.4^{\# \#}$ & $57.9 \pm 40.8^{\# \# \#}$ & $52.2 \pm 39.4^{\#}$ \\
\hline \multicolumn{6}{|l|}{ Education (\%) } \\
\hline$<9$ th grade & $16 \%$ & $9 \%$ & $10 \%$ & $6 \%$ & $11 \%$ \\
\hline $9-11$ th grade & $18 \%$ & $13 \%$ & $14 \%$ & $14 \%$ & $17 \%$ \\
\hline Graduate high school & $24 \%$ & $26 \%$ & $21 \%$ & $19 \%$ & $19 \%$ \\
\hline Some college & $27 \%$ & $32 \%$ & $27 \%$ & $31 \%$ & $36 \%$ \\
\hline \multirow[t]{4}{*}{$\begin{array}{l}\text { Graduated 4-yr } \\
\text { college }\end{array}$} & $14 \%$ & $17 \%$ & $27 \%$ & $29 \%$ & $17 \%$ \\
\hline & \multicolumn{5}{|c|}{ Men } \\
\hline & Q1 & Q2 & Q3 & Q4 & Q5 \\
\hline & $(\mathrm{n}=487)$ & $(n=475)$ & $(n=477)$ & $(n=480)$ & $(\mathrm{n}=470)$ \\
\hline $\begin{array}{l}\text { Physical Activity } \\
\text { (METmin/week) } \dagger\end{array}$ & $7 \pm 26$ & $709 \pm 352^{\# \#}$ & $2288 \pm 616^{\# \# \#}$ & $5651 \pm 1488^{\# \# \#}$ & $16269 \pm 7255^{\# \# \#}$ \\
\hline Age (y) & $48.4 \pm 14.8$ & $44.7 \pm 15.2^{\# \#}$ & $41.8 \pm 14.8^{\# \#}$ & $39.0 \pm 14.9^{\# \#}$ & $38.8 \pm 13.7^{\# \#}$ \\
\hline Body Weight (kg) & $87.2 \pm 17.8$ & $85.7 \pm 16.6$ & $85.4 \pm 16.7$ & $87.0 \pm 16.6$ & $84.0 \pm 15.1^{\# \#}$ \\
\hline $\begin{array}{l}\text { Body Mass Index } \\
\left(\mathrm{kg} / \mathrm{m}^{2}\right)\end{array}$ & $28.8 \pm 4.9$ & $28.0 \pm 4.7^{\# \#}$ & $27.5 \pm 4.6^{\# \#}$ & $28.1 \pm 4.7^{\#}$ & $27.5 \pm 4.6^{\# \#}$ \\
\hline $\begin{array}{l}\text { Household Income } \\
(\$ 1000)\end{array}$ & $49.6 \pm 36.8$ & $61.0 \pm 42.7^{\# \# \#}$ & $64.5 \pm 41.9^{\# \# \#}$ & $57.4 \pm 39.4^{\#}$ & $50.9 \pm 34.4$ \\
\hline \multicolumn{6}{|l|}{ Education (\%) } \\
\hline$<9$ th grade & $17 \%$ & $10 \%$ & $8 \%$ & $8 \%$ & $12 \%$ \\
\hline $9-11$ th grade & $21 \%$ & $16 \%$ & $15 \%$ & $15 \%$ & $19 \%$ \\
\hline Graduate high school & $25 \%$ & $21 \%$ & $19 \%$ & $28 \%$ & $31 \%$ \\
\hline Some college & $22 \%$ & $25 \%$ & $26 \%$ & $30 \%$ & $27 \%$ \\
\hline $\begin{array}{l}\text { Graduated 4-yr } \\
\text { college }\end{array}$ & $14 \%$ & $27 \%$ & $31 \%$ & $18 \%$ & $9 \%$ \\
\hline
\end{tabular}

\subsection{Relationship between Physical Activity and Sugar Intake}

As shown in Figure 1, there was a curvilinear relationship with habitual PA for intakes of added sugar from sugary foods as well as sugar from sugar-sweetened beverages. In women, added sugar intake from sugary foods was significantly lower in moderately active $(\mathrm{Q} 2:-2 \pm 1$ tsp/day, $\mathrm{p}=0.03, \mathrm{~d}=0.15 ; \mathrm{Q} 3:-2 \pm 1 \mathrm{tsp} / \mathrm{day}, \mathrm{p}=0.005, d=0.19)$ and highly active individuals $(\mathrm{Q} 4:-2 \pm 1$ 
tsp/day, $\mathrm{p}=0.005, d=0.18)$ when compared to least active individuals ( $\mathrm{Q} 1: 20 \pm 1$ tsp/day), whereas added sugar intake from sugary foods in the highest activity quintile (Q5) was not significantly different from Q1 $(\mathrm{p}=0.52)$. In men, added sugar intake from sugary foods did not differ significantly between least active (Q1) and moderately active (Q2-Q3) and highly active individuals $(\mathrm{Q} 4)$, but was significantly elevated in the highest activity quintile $(\mathrm{Q} 5:+3 \pm 1$ tsp/day, $\mathrm{p}=0.007, d=0.21)$ when compared to $\mathrm{Q} 1$ ( $30 \pm 1$ tsp/day).

Similarity, sugar from sugar-sweetened beverages (not shown) was significantly lower in women in Q2 ( $-2 \pm 1$ tsp/day, $\mathrm{p}=0.02, d=0.17), \mathrm{Q} 3(-2 \pm 1$ tsp/day, $\mathrm{p}=0.01, d=0.17)$, and $\mathrm{Q} 4(-2 \pm 1$ tsp/day, $\mathrm{p}=0.009, d=0.17)$ when compared to $\mathrm{Q} 1(16 \pm 1$ tsp/day). In men, sugar from sugarsweetened beverages was significantly lower in Q3 ( $-3 \pm 1$ tsp/day, $\mathrm{p}=0.02, d=0.15)$ when compared to Q1 ( $26 \pm 1 \mathrm{tsp} /$ day). The contribution of sugar from sugar-sweetened beverages to total added sugar intake (Figure 1 right) was significantly reduced in moderately (Q3: $-7.5 \pm$ $2.0 \%, \mathrm{p}<0.001, d=0.26)$ and highly active women $(\mathrm{Q} 4:-5.4 \pm 2.0 \%, \mathrm{p}=0.006, d=0.19)$ when compared to the lowest activity quintile $(\mathrm{Q} 1: 62.0 \pm 2.6 \%)$. In men, the contribution of sugar from sugar-sweetened beverages decreased significantly from the lowest (Q1: 74.5 $\pm 2.7 \%$ ) to moderate activity levels $(\mathrm{Q} 3:-5.5 \pm 2.1 \%, \mathrm{p}=0.01, d=0.17)$ before increasing again and exceeding Q1 in the highest activity quintile $(\mathrm{Q} 5:+5.7 \pm 2.1 \%, \mathrm{p}=0.008, d=0.21)$.

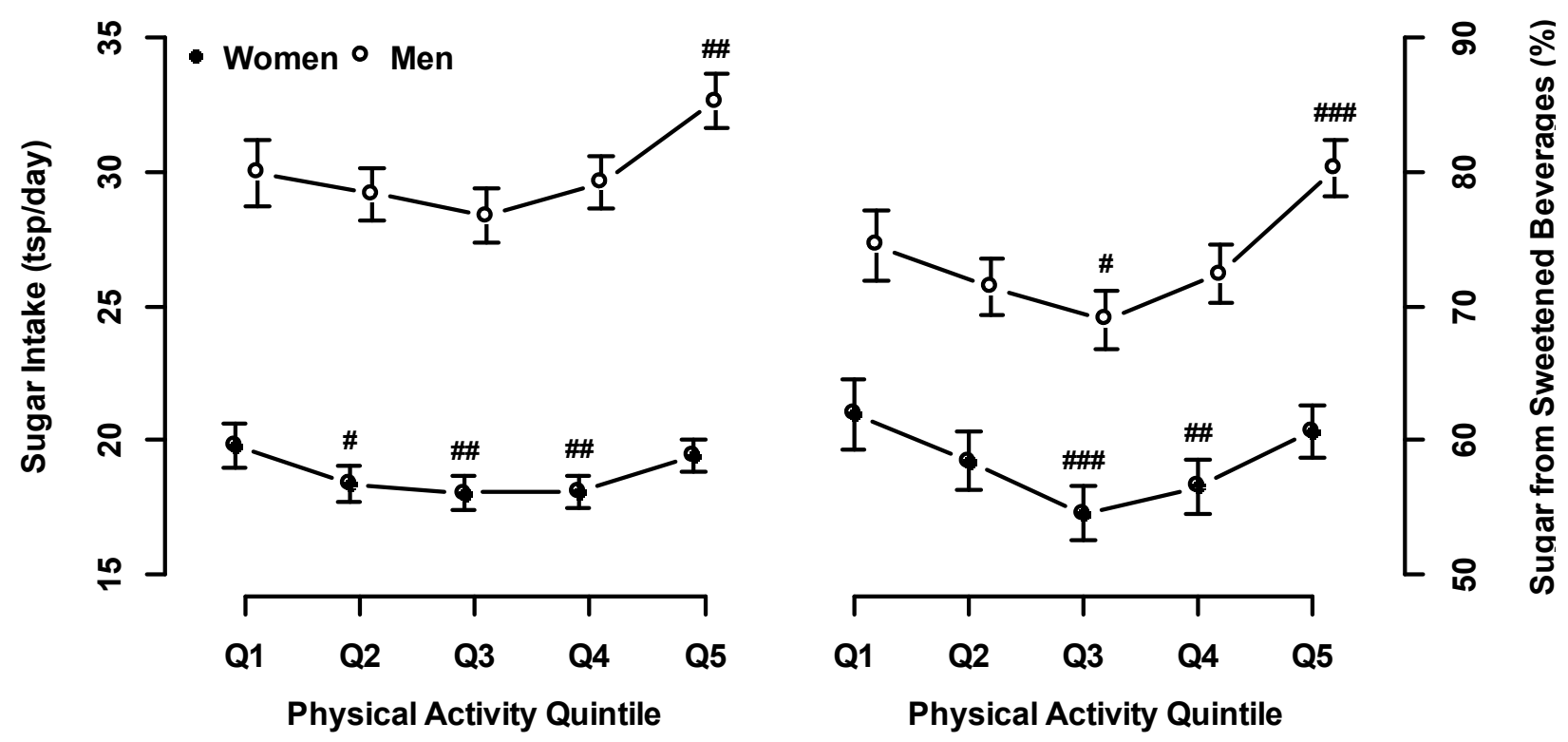

Figure 1 Model estimates of sugar intake (left) and sugar intake from sugar-sweetened beverages (right) relative to physical activity levels in women (closed symbols) and men (open symbols). Error bars indicate standard error of the estimate. Intakes are adjusted for age, BMI, income, and education level; \#, \#\#, \#\#\#: different from Q1 ( $p<0.05, p<0.01, p<0.001$, respectively).

3.3. Relationship between Physical Activity and Fruits \& Vegetables, Fiber, Whole Grains and Dairy Intake

As shown in Figure 2 and Figure 3, the intake of fruits and vegetables, fiber, whole grains and dairy increased gradually with increasing PA levels, but plateaued at the highest activity levels 
for almost all variables. In women, fruit and vegetable intake increased gradually from low to moderate $(\mathrm{Q} 3:+0.2 \pm 0.1$ cup equivalents; $\mathrm{p}=0.03, d=0.15)$ and high activity levels $(\mathrm{Q} 4:+0.3 \pm$ 0.1 cup equivalents, $\mathrm{p}<0.001, d=0.29)$ and remained high in the highest activity quintile (Q5: $+0.3 \pm 0.1$ cup equivalents, $\mathrm{p}<0.001, d=0.28$ ) when compared to $\mathrm{Q} 1$ ( $1.9 \pm 0.1$ cup equivalents). In men, fruit and vegetable intake was significantly elevated in Q3 $(+0.3 \pm 0.1$ cup equivalents, $\mathrm{p}=0.002, \mathrm{~d}=0.23)$ and $\mathrm{Q} 5(+0.2 \pm 0.1$ cup equivalents, $\mathrm{p}=0.01, d=0.19)$ when compared to Q1 ( $2.4 \pm 0.1$ cup equivalents). Fiber intake increased consistently from the least active women (Q1: $12 \pm 1 \mathrm{~g}$ ) to the most active women (Q4: $+2 \pm 0, \mathrm{p}<0.001, d=0.25$; Q5: $+1 \pm 0 \mathrm{~g}, \mathrm{p}<0.001$, $d=0.23)$. In men, fiber intake was significantly elevated over Q1 $(18 \pm 1 \mathrm{~g})$ in moderately (Q3: $+2 \pm 1 \mathrm{~g}, \mathrm{p}=0.001, d=0.24)$, and most active men $(\mathrm{Q} 4:+1 \pm 1 \mathrm{~g}, \mathrm{p}=0.04, d=0.16 ; \mathrm{Q} 5(+2 \pm 1 \mathrm{~g}$, $\mathrm{p}=0.003, d=0.23)$. Whole grain intake was significantly elevated in $\mathrm{Q} 4$ in both women $(+0.3 \pm$ 0.1 ounce equivalents, $\mathrm{p}=0.03, d=0.15)$ and men $(+0.3 \pm 0.1$ ounce equivalents, $\mathrm{p}=0.03, d=0.15)$. Dairy intake differed across the activity spectrum only in men, but not in women. In men, dairy intake was significantly elevated in Q2 $(+0.2 \pm 0.1$ cup equivalents, $\mathrm{p}=0.02, d=0.17), \mathrm{Q} 3(+0.3 \pm$ 0.1 cup equivalents, $\mathrm{p}<0.001, d=0.26)$, $\mathrm{Q} 4(+0.3 \pm 0.1$ cup equivalents, $\mathrm{p}<0.001, d=0.28)$, and $\mathrm{Q} 5(+0.3 \pm 0.1$ cup equivalents, $\mathrm{p}<0.001, d=0.30)$ when compared to Q1 $(1.5 \pm 0.1$ cup equivalents).
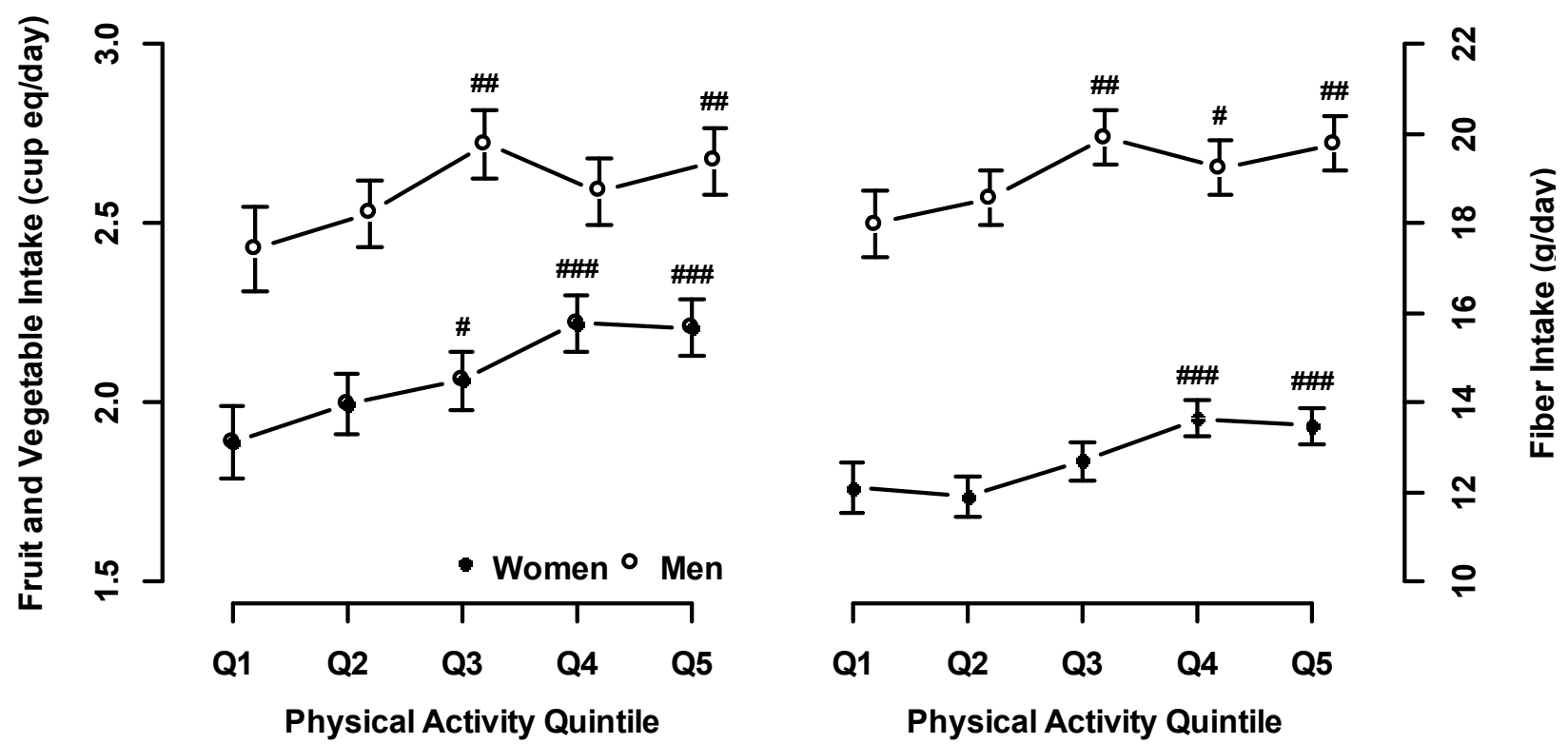

Figure 2 Model estimates of fruit and vegetable intake (left) and fiber intake (right) relative to physical activity levels in women (closed symbols) and men (open symbols). Error bars indicate standard error of the estimate. Intakes are adjusted for age, BMI, income, and education level; \#, \#\#, \#\#\#: different from Q1 ( $p<0.05, p<0.01, p<0.001$, respectively). 

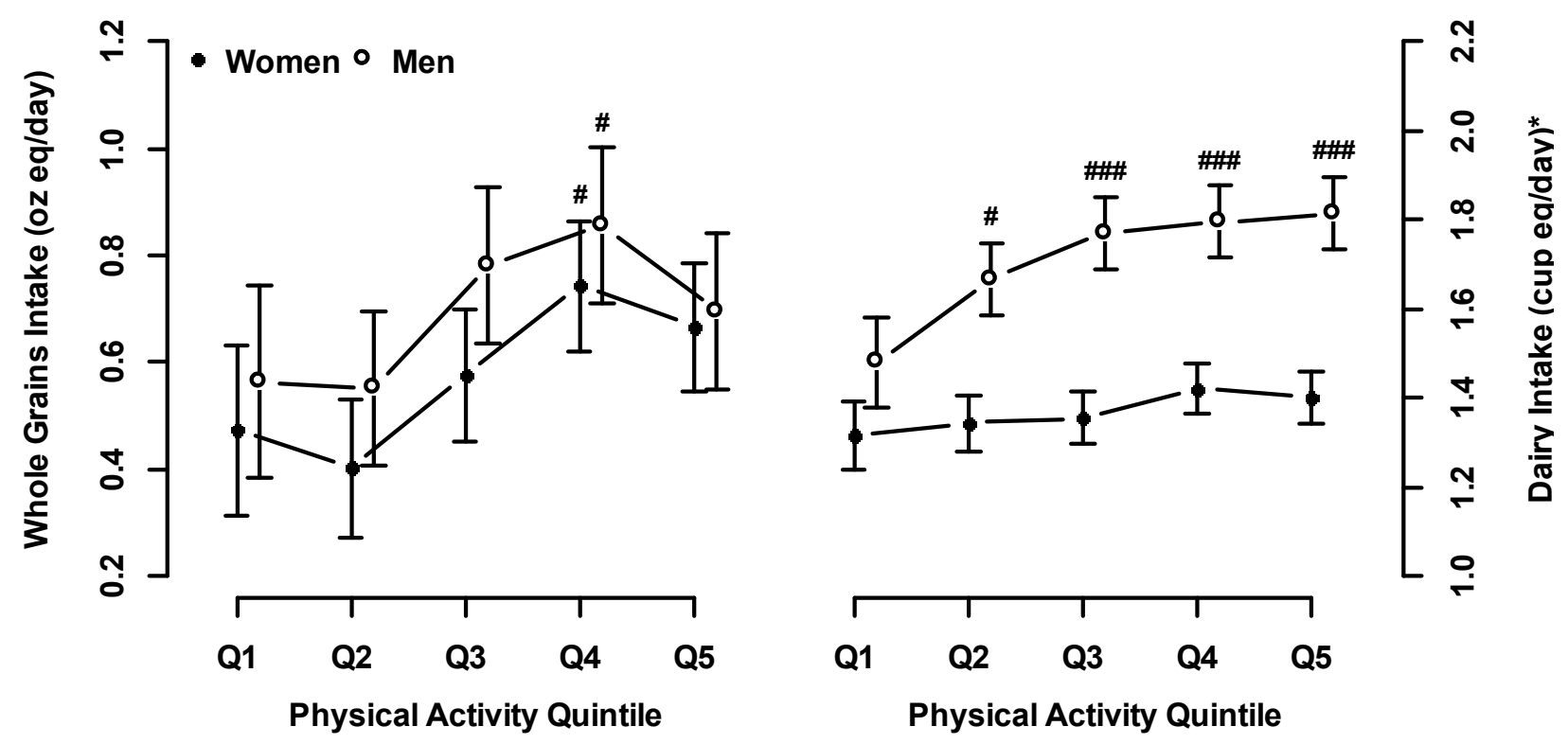

Figure 3 Model estimates of whole grain intake (left) and dairy intake (right) relative to physical activity levels in women (closed symbols) and men (open symbols). Error bars indicate standard error of the estimate. Intakes are adjusted for age, BMI, income, and education level; \#, \#\#, \#\#\#: different from Q1 $(p<0.05, p<0.01, p<0.001$, respectively).

\section{Discussion}

Our results demonstrate a differential relationship between habitual PA and the consumption of key dietary factors associated with food choices: Individuals who are moderately to highly physically active tend to consume less energy-dense, nutrient-poor foods - such as added sugar from sugary foods and sugar from sugar-sweetened beverages - and ingest more nutrient-dense food, such as fruits, vegetables, whole grains, and dairy. However, the intakes of added sugar from sugary foods and sugar from sugar-sweetened beverages increase again towards the upper end of the activity spectrum, whereas intakes of healthier food items - such as fruits, vegetables, whole grains, and dairy - do not. Taken together, these findings suggests that the beneficial effects of PA on eating patterns only exist for the transition from low to moderate and high PA and are inhibited at very high levels of PA. These opposing trends for intakes of energy-dense and nutrient-dense foods provide further evidence that PA does not modulate food intake simply in terms of overall intake, but selectively impacts choices of foods with various nutritive properties.

Our first important finding of the relationship between PA and added sugar from sugary foods intake is in striking resemblance with the previously reported relationship between habitual PA and overall dietary EI. Previously established in rodents (40), a curvilinear relationship was first reported in Indian factory workers, in whom dietary EIs were highest among inactive (stallholders, supervisors) and highly active workers (coalmen, blacksmiths, carriers) and were lower among moderately active workers (clerks, mechanics, drivers) (13). It was noteworthy that the excessive EI only had implications for body weight in the inactive workers, who were on average about 25 pounds $(22 \%)$ heavier than active workers, whereas body weight remained fairly stable as dietary EI transitioned from moderate to high activity levels (13). As additional 
evidence of this curvilinear relationship emerged, Blundell et al. deemed the lower end of the activity spectrum the "unregulated zone", in which body weight increases in response to the increased EI from a moderately active to an inactive lifestyle (12). In contrast, in the "regulated zone", which refers to the upper end of the activity spectrum, body mass remains stable despite an increase in EI, suggesting that the higher EI is reflective of compensatory mechanisms to meet increased energy needs in order to maintain body weight stability (12). The implications of this differential association between activity level, dietary intake, and body weight regulation was also highlighted in a recent prospective study by Shook et al., which revealed that increased dietary EI was only associated with an increased risk of unhealthy weight gain in the unregulated (inactive) zone, but not in the regulated (highly active) zone (16). Although not the primary purpose of our analyses, our data provide further confirmation of the implications for body weight regulation as demonstrated by a 5\%-reduction in body mass index from Q1-Q3 in both sexes, followed by stagnating BMIs despite increases in added sugar intake from Q3-Q5.

Our results further highlight the contribution of added sugar, an energy-dense, nutrient-poor food item, contributing to overall EI at opposite ends of the PA spectrum. Specifically, a recent systematic review linked increased inactive behavior with greater consumption of sugarsweetened beverages (41). In contrast, higher levels of PA have been associated with increased consumption of sport and energy drinks (42), suggesting that the high intake of added sugar in highly active individuals may be reflective of increased carbohydrate requirements in individuals who conduct prolonged aerobic activities (43).

Our finding of a non-linear relationship between PA and added sugar intake may also explain why previous literature has been inconclusive about dietary differences between active and inactive individuals. For example, a recent systematic review concluded that increased PA and exercise have no consistent effect on energy or macronutrient intake, with the exception of a lower fat intake with increased PA (44). As such, future studies linking PA and eating patterns should carefully account for these non-linear patterns between inactive, moderately active, and highly active individuals.

We further demonstrate increased intakes of nutrient-dense food items, including fruits and vegetables, whole grains, fiber, and dairy, as individuals progress from an inactive to a moderately active lifestyle, which is in support of previous literature. In the Bogalusa Heart study, consumption of fruit and dairy was higher and fast food and fat consumption was lower among highly active when compared to the least active individuals $(17,19)$. Unfortunately, this study was conducted only in young adults (average age $29 \mathrm{y}$ ) and PA levels were only assessed as a self-report on a 5-item Likert ranging from physically inactive to very active. In another study comparing young exercisers to non-exercisers, exercisers were more likely to meet the recommended intakes for grain and fruit intake (20). As such, our results confirm these findings in a large-scale, representative data set across all adult age groups. In addition, the plateau at the upper end of the activity spectrum that was consistently observed in intakes of all nutrient-dense food items highlights the need to address non-linear effects between PA and food choices. It is noteworthy that an increase in dairy intake with increasing PA was only observed in men but not in women. While there is only limited comparable literature available, this finding is in agreement with previous data suggesting that sports and exercise participation had a positive influence on dairy consumption in male but not in female adolescent (45). Considering that men are more likely to engage in muscle building activities, whereas women tend to conduct more 
aerobic exercise with an emphasis on weight management (46), and that dairy consumption is often implicated in strength and muscle building activities (47), this sex discrepancy does not seem surprising.

While several studies, including our own, have reported a similar shaped relationship between PA and dietary intakes, the approach to quantify PA varied. In the present study, we chose to express PA in MET-minutes, a simple physiological measure of the energy expended during PA (48), to account for the higher energy cost of vigorous when compared to moderate activity. Although Cantennaci et al. (2014) also used energy expenditure, expressed in kcal/week, to assign participants in the National Weight Loss Registry into different activity groups, their approach was ultimately anchored around time spent during PA, ranging from $<30$ minutes/day in the lowest to $>90$ minutes/day in the highest group (14). A similar approach was also adopted by Shook and colleagues, who divided participants into quintiles based on their time spent during moderate-to-vigorous PA, ranging from 15.7 minutes/day in the lowest to 174.5 minutes/day in the highest quintile (16). In their systematic review, Beaulieu et al. (2016) used a combination of various approaches, including time spent and energy expended during PA as well as overall PA level (PAL), defined as total daily energy expenditure over resting energy expenditure, to divide their participants into four levels of activity, ranging from low $(<150$ minutes/week; $<1000 \mathrm{kcal} /$ week; PAL $=1.4-1.69)$ to very high ( $>840$ minutes/week; $>3500 \mathrm{kcal} / \mathrm{week})(15)$. Regardless of these methodological differences, our activity quintiles, when converted into time spent during PA, are strikingly similar to the levels defined by others, which is even more remarkable considering that other data sets were not sex balanced (e.g. (14)), included only participants from a specific age group (16), or combined data from multiple studies (15).

Despite these similarities in our groupings, it should be noted that PA was collected via selfreport using the GPAQ (34), while others have used objective methods to quantify PA. For example, Shook et al. (16) utilized the SenseWear armband, which combines three-dimensional accelerometry with other biosensors related to sweat rate and heat production (49) to objectively quantify time spent in moderate-to-vigorous activities. Although accelerometry has been used in previous and subsequent iterations of the NHANES, PA was not measured objectively during the 2009-2010 NHANES, the first time the DSQ was employed (33). The use of accelerometry would have allowed us to factor in the contribution of light PA to total PA, particularly in those reporting no moderate or vigorous PA at all. The GPAQ itself did not overestimate the proportion of the sample who did not conduct any moderate or vigorous PA, which was fairly similar in our sample (23\% overall) when compared to accelerometer-based measurements $(29.7 \%)$ in a nationally representative sample (50). However, the fact that only 3 of the 14 studies included in the systematic review by Beaulieu et al. used objectively measured PA highlights the need for objective PA assessment in future studies.

While the development of the DSQ aimed to improve dietary data assessment by using larger time frames ( 30 days versus 1 day) and providing outcome variables that can be used for actionable recommendations (e.g., individuals can explore healthier food choices rather than nutrient choices), the DSQ continues to rely on self-reported data, a topic that is discussed widely in the scientific community (51). Despite obvious limitations, self-report remains the most advanced method available for detecting population-level dietary patterns and can be used successfully to inform dietary guidance and public health policy (52). While our future work 
aims to utilize biomarkers (53), this method would be costly and time consuming without first conducting a secondary data analysis to support this future work.

Although our analysis demonstrates significant changes intake of key dietary factors relative to PA, we employed a linear model approach to compare intake in higher activity quintiles to the lowest quintile of inactive individuals, which served as reference quintile. As a result, there is only direct statistical evidence for a curvilinear relationship between habitual PA and the percentage of sugar from sugar-sweetened beverage, which was - when compared to the reference quintile - significantly reduced in Q3 and significantly elevated in Q5. Likewise, for nutrient-dense foods, the comparison between the reference quintile and higher PA quintiles for nutrient-dense foods revealed significant increases, although the linear model did not allow us to test for a ceiling effect. However, graphical inspection of the data, combined with the relative large sample size and the presence of null-findings (e.g., no significant difference in women between Q1 and Q5 for added sugar) indirectly support our assumptions. Given the expected differential changes between inactivity and moderate PA levels for added sugar (decreasing) and nutrient-dense foods (increasing), we utilized the same statistical approach for all variables, and the use of linear modeling allowed us to directly quantify differences in dietary factors relative to Q1. A further limitation of the present study is its cross-sectional nature. As such, it remains to be explored whether the curvilinear relationship between habitual PA and food intake is upheld in individuals who alter their PA status to transition from an unregulated to a regulated activity zone. In fact, there is ample evidence that an individual's food intake response in an exercise program aimed at increasing energy expenditure can vary substantially (12), and that this interindividual variability in food intake may determine the success of weight loss (54).

\section{Conclusions}

Our results demonstrate a differential relationship between habitual PA and eating patterns and nutrient intakes, indicating that the increased EI observed in individuals at the lower and upper end of the PA spectrum is at least partially explained by an increased intake of added sugar from sugary foods and sugar-sweetened beverages and a (relatively) lower intake of nutrient-dense foods which typically are less-energy dense. More rigorous prospective experiments are needed to test whether this differential relationship between PA and dietary intakes persists as individuals alter their PA habits. Future research is further needed to examine specific mechanisms of food choices among those engaging in various levels of PA in order to ensure the dietary behaviors (i.e., increased sugary food intake) do not negate the positive effects of physical activity, such as decreased risk for obesity and chronic diseases such as heart disease, type 2 diabetes, and certain cancers (3-5).

\section{Declarations of interests:}

The authors declare they have no competing interest to disclose.

\section{Acknowledgements:}

This work was supported by a Food for Health Collaboration Initiative grant from the University of Nebraska. 


\section{References}

1. Ogden CL, Carroll MD, Kit BK, Flegal KM. Prevalence of obesity among adults: United States, 2011-2012. NCHS Data Brief. 2013(131):1-8.

2. Romieu I, Dossus L, Barquera S, Blottière HM, Franks PW, Gunter M, et al. Energy balance and obesity: what are the main drivers? Cancer Causes \& Control. 2017;28(3):247-58.

3. Cornelissen VA, Smart NA. Exercise training for blood pressure: a systematic review and meta-analysis. J Am Heart Assoc. 2013;2(1):e004473.

4. Goodyear LJ, Kahn BB. Exercise, glucose transport, and insulin sensitivity. Annu Rev Med. 1998;49:235-61.

5. Moore SC, Lee I, Weiderpass E, et al. Association of leisure-time physical activity with risk of 26 types of cancer in 1.44 million adults. JAMA Internal Medicine. 2016;176(6):816-25.

6. Dombrowski SU, Knittle K, Avenell A, Araujo-Soares V, Sniehotta FF. Long term maintenance of weight loss with non-surgical interventions in obese adults: systematic review and meta-analyses of randomised controlled trials. BMJ. 2014;348:g2646.

7. Caudwell P, Gibbons C, Finlayson G, Näslund E, Blundell J. Physical Activity, Energy Intake, and Obesity: The Links Between Exercise and Appetite. Current Obesity Reports. 2013;2(2):185-90.

8. Andrew P, Susan J. Energy Intake/Physical Activity Interactions in the Homeostasis of Body Weight Regulation. Nutr Rev. 2004;62(s2):S98-S104.

9. Hill JO, Wyatt HR, Peters JC. Energy balance and obesity. Circulation. 2012;126(1):126-

32.

10. Hopkins M, Blundell JE. Energy balance, body composition, sedentariness and appetite regulation: pathways to obesity. Clin Sci (Lond). 2016;130(18):1615-28.

11. King NA, Caudwell PP, Hopkins M, Stubbs JR, Naslund E, Blundell JE. Dual-process action of exercise on appetite control: increase in orexigenic drive but improvement in mealinduced satiety. The American journal of clinical nutrition. 2009;90(4):921-7.

12. Blundell JE. Physical activity and appetite control: can we close the energy gap? Nutrition Bulletin. 2011;36(3):356-66.

13. Mayer J, Roy P, Mitra KP. Relation between Caloric Intake, Body Weight, and Physical Work: Studies in an Industrial Male Population in West Bengal. The American Journal of Clinical Nutrition. 1956;4(2):169-75.

14. Catenacci VA, Odgen L, Phelan S, Thomas JG, Hill J, Wing RR, et al. Dietary Habits and Weight Maintenance Success in High Versus Low Exercisers in the National Weight Control Registry. Journal of Physical Activity and Health. 2014;11(8):1540-8.

15. Beaulieu K, Hopkins M, Blundell J, Finlayson G. Does Habitual Physical Activity Increase the Sensitivity of the Appetite Control System? A Systematic Review. Sports Med. 2016;46(12):1897-919.

16. Shook RP, Hand GA, Drenowatz C, Hebert JR, Paluch AE, Blundell JE, et al. Low levels of physical activity are associated with dysregulation of energy intake and fat mass gain over 1 year. The American Journal of Clinical Nutrition. 2015;102(6):1332-8.

17. Deshmukh-Taskar P, Nicklas TA, Yang S-J, Berenson GS. Does Food Group Consumption Vary by Differences in Socioeconomic, Demographic, and Lifestyle Factors in Young Adults? The Bogalusa Heart Study. Journal of the American Dietetic Association. 2007;107(2):223-34.

18. Russell J, Tom B, Sunmi Y, W. CK, Issa Z, Kathy W, et al. Relationship between Physical Activity and Diet among African-American Girls. Obes Res. 2004;12(S9):55S-63S. 
19. Jago R, Nicklas T, Yang SJ, Baranowski T, Zakeri I, Berenson GS. Physical activity and health enhancing dietary behaviors in young adults: Bogalusa Heart Study. Prev Med. 2005;41(1):194-202.

20. Georgiou C, Betts N, Hoos T, Glenn M. Young Adult Exercisers and Nonexercisers Differ in Food Attitudes, Perceived Dietary Changes, and Food Choices. International Journal of Sport Nutrition. 1996;6(4):402-13.

21. Westerterp KR. Physical activity and physical activity induced energy expenditure in humans: measurement, determinants, and effects. Front Physiol. 2013;4:90.

22. Centers for Disease Control and Prevention. National Center for Chronic Disease Prevention and Health Promotion DoN, Physical Activity, and Obesity. Data, Trend and Maps 2018 [Available from: https://www.cdc.gov/nccdphp/dnpao/data-trends-maps/index.html.

23. Centers for Disease Control and Prevention. National Center for Health Statistics. Health, United States, 2017 - $\quad$ Data Finder [Available from: https://www.cdc.gov/nchs/hus/contents2017.htm?search=Physical activity/inactivity.

24. Udo T, Grilo CM. Prevalence and Correlates of DSM-5-Defined Eating Disorders in a Nationally Representative Sample of U.S. Adults. Biological Psychiatry. 2018;84(5):345-54.

25. Office of Disease Prevention and Health Promotion. 2015-2020 Dietary Guidelines for Americans [Available from: https://health.gov/dietaryguidelines/2015/.

26. Catenacci VA, Odgen L, Phelan S, Thomas JG, Hill J, Wing RR, et al. Dietary habits and weight maintenance success in high versus low exercisers in the National Weight Control Registry. J Phys Act Health. 2014;11(8):1540-8.

27. Thompson FE, Byers T. Dietary assessment resource manual. J Nutr. 1994;124(11 Suppl):2245S-317S.

28. Yaroch AL, Byker C, Pinard CA, Smith TM. Advances in methodologies for assessing dietary intake and physical activity among adolescents. Adolesc Med State Art Rev. 2012;23(3):610-30.

29. National Cancer Institute. Screeners at a Glance. Dietary Assessment Primer. [Available from: https://dietassessmentprimer.cancer.gov/profiles/screeners/.

30. Dhurandhar NV, Schoeller D, Brown AW, Heymsfield SB, Thomas D, Sorensen TI, et al. Energy balance measurement: when something is not better than nothing. Int $\mathrm{J}$ Obes (Lond). 2015;39(7):1109-13.

31. Kim DJ, Holowaty EJ. Brief, validated survey instruments for the measurement of fruit and vegetable intakes in adults: a review. Prev Med. 2003;36(4):440-7.

32. Yaroch AL, Tooze J, Thompson FE, Blanck HM, Thompson OM, Colon-Ramos U, et al. Evaluation of three short dietary instruments to assess fruit and vegetable intake: the National Cancer Institute's food attitudes and behaviors survey. J Acad Nutr Diet. 2012;112(10):1570-7.

33. Thompson FE, Midthune D, Kahle L, Dodd KW. Development and Evaluation of the National Cancer Institute's Dietary Screener Questionnaire Scoring Algorithms. J Nutr. 2017;147(6):1226-33.

34. Armstrong T, Bull F. Development of the World Health Organization Global Physical Activity Questionnaire (GPAQ). Journal of Public Health. 2006;14(2):66-70.

35. Hills AP, Mokhtar N, Byrne NM. Assessment of Physical Activity and Energy Expenditure: An Overview of Objective Measures. Frontiers in Nutrition. 2014;1(5).

36. Craig CL, Marshall AL, Sjostrom M, Bauman AE, Booth ML, Ainsworth BE, et al. International physical activity questionnaire: 12-country reliability and validity. Med Sci Sports Exerc. 2003;35(8):1381-95. 
37. Ainsworth BE, Haskell WL, Herrmann SD, Meckes N, Bassett DR, Tudor-Locke C, et al. 2011 Compendium of Physical Activities: a second update of codes and MET values. Medicine and science in sports and exercise. 2011;43(8):1575-81.

38. World Health Organization. Global Physical Activity Questionnaire Analysis Guide. www.who.int/chp/steps/resources/GPAQ Analysis_Guide.pdf?ua=1.

39. Saffer H, Dave D, Grossman M, Leung LA. Racial, Ethnic, and Gender Differences in Physical Activity. Journal of human capital. 2013;7(4):378-410.

40. Mayer J, Marshall NB, Vitale JJ, Christensen JH, Mashayekhi MB, Stare FJ. Exercise, Food Intake and Body Weight in Normal Rats and Genetically Obese Adult Mice. American Journal of Physiology-Legacy Content. 1954;177(3):544-8.

41. Hobbs M, Pearson N, Foster PJ, Biddle SJ. Sedentary behaviour and diet across the lifespan: an updated systematic review. Br J Sports Med. 2015;49(18):1179-88.

42. Larson N, Dewolfe J, Story M, Neumark-Sztainer D. Adolescent consumption of sports and energy drinks: linkages to higher physical activity, unhealthy beverage patterns, cigarette smoking, and screen media use. J Nutr Educ Behav. 2014;46(3):181-7.

43. Burke LM, Loucks AB, Broad N. Energy and carbohydrate for training and recovery. J Sports Sci. 2006;24(7):675-85.

44. Donnelly JE, Herrmann SD, Lambourne K, Szabo AN, Honas JJ, Washburn RA. Does increased exercise or physical activity alter ad-libitum daily energy intake or macronutrient composition in healthy adults? A systematic review. PLoS One. 2014;9(1):e83498.

45. Racey M, Bransfield J, Capello K, Field D, Kulak V, Machmueller D, et al. Barriers and Facilitators to Intake of Dairy Products in Adolescent Males and Females With Different Levels of Habitual Intake. Global Pediatric Health. 2017;4:2333794X17694227.

46. Bryan AD, Rocheleau CA. Predicting aerobic versus resistance exercise using the theory of planned behavior. Am J Health Behav. 2002;26(2):83-94.

47. Tunick MH, Van Hekken DL. Dairy Products and Health: Recent Insights. J Agric Food Chem. 2015;63(43):9381-8.

48. Bushman BA. Wouldn't You Like to Know: How Can I Use METs to Quantify the Amount of Aerobic Exercise? ACSM's Health \& Fitness Journal. 2012;16(2):5-7.

49. Koehler K, Drenowatz C. Monitoring Energy Expenditure Using a Multi-Sensor Device -Applications and Limitations of the SenseWear Armband in Athletic Populations. Front Physiol. 2017;8(983).

50. Evenson KR, Wen F, Metzger JS, Herring AH. Physical activity and sedentary behavior patterns using accelerometry from a national sample of United States adults. International Journal of Behavioral Nutrition and Physical Activity. 2015;12(1):20.

51. Subar AF, Freedman LS, Tooze JA, Kirkpatrick SI, Boushey C, Neuhouser ML, et al. Addressing Current Criticism Regarding the Value of Self-Report Dietary Data. J Nutr. 2015;145(12):2639-45.

52. Davy BM, Estabrooks PA. The Validity of Self-reported Dietary Intake Data: Focus on the "What We Eat In America" Component of the National Health and Nutrition Examination Survey Research Initiative. Mayo Clin Proc. 2015;90(7):845-7.

53. Naska A, Lagiou A, Lagiou P. Dietary assessment methods in epidemiological research: current state of the art and future prospects. F1000Res. 2017;6:926.

54. King NA, Hopkins M, Caudwell P, Stubbs RJ, Blundell JE. Individual variability following 12 weeks of supervised exercise: identification and characterization of compensation for exercise-induced weight loss. Int J Obes (Lond). 2008;32(1):177-84. 